\title{
La planificación estratégica como mejora del desempeño docente en Perú
}

\author{
Strategic Planning as an improvement in teaching performance in Perú \\ O planejamento estratégico como uma melhoria no desempenho docente no Perú
}

Stephanie Marjorie Valladares-Durand ${ }^{1}$

stephanie.valladares@unmsm.edu.pe

https://orcid.org/0000-00001-8187-0224

Colbert Martin Soto-Rivera ${ }^{2}$

csotor@cientifica.edu.pe.edu.pe

https://orcid.org/0000-0003-0743-7072
Amilcar Máximo Vicuña-Ureta
avicunau@unmsm.edu.pe
https://orcid.org/0000-0003-1548-2252

Gleny Secibel Jara-Llanos $^{3}$
gjara@une.edu.pe
https://orcid.org/0000-0002- 9284-7545

${ }^{1}$ Universidad Nacional Mayor de San Marcos, Lima-Perú

${ }^{2}$ Universidad Científica del Sur, Lima-Perú

${ }^{3}$ Universidad Nacional Enrique Guzmán y Valle. Lima-Perú

Artículo recibido 4 de noviembre 2021, arbitrado y aceptado 7 de diciembre 2021 y publicado 7 de marzo 2022

\section{ABSTRACT}

Las instituciones educativas, igual que las empresas, deben deposeer una organización interna que les permita capitalizar sus fuerzas hacia objetivos específicos; sin embargo, la coyuntura puede modificar las expectativas y estrategias a emplear. Así, todas las instituciones deben tener una planificación estratégica que alinee a los docentes, comprendiendo que la buena gestión educativa garantiza el éxito de toda la comunidad. Por ello, el objetivo de este estudio es dilucidar el impacto que tiene una buena planificación estratégica en la mejora del desempeño de los docentes del Perú, tomando en consideración: sus características, beneficios y procesos. Para este fin, se realizó una revisión sistemática exploratoria y análisis datos. Finalmente, gracias a la rigurosa selección de información se pudo concluir que, en efecto, existe una relación directamente proporcional entre planificación estratégica y desempeño docente, expresado en la mejora de tiempo, orden y ejecución.

Palabras clave: Estrategias educativas; Planificación; Competencia profesional; Gestión; aprendizaje
RESUMEN

Educational institutions, like companies, must have an internal organization that allows them to capitalize on their strength towards specific objectives; however, the situation can modify the expectations and strategies to be used. Thus, all institutions must have strategic planning that aligns teachers, understanding that good educational management guarantees the success of the entire community. Therefore, the objective of this study is to elucidate the impact that good strategic planning has on improving the performance of teachers in Peru, taking into consideration: their characteristics, benefits and processes. For this purpose, an exploratory systematic review and data analysis was performed. Finally, thanks to the rigorous selection of information, it was possible to conclude that, in effect, there is a directly proportional relationship between strategic planning and teaching performance, expressed in the improvement of time, order and execution.

Key words: Educational strategies; Planning; Occupational qualifications; Management; learning

\section{RESUMO}

As instituições educacionais, como as empresas, devem ter uma organização interna que lhes permita capitalizar sua força para objetivos específicos; entretanto, a situação pode modificar as expectativas e estratégias a serem utilizadas. Assim, todas as instituições devem ter um planejamento estratégico que alinhe os professores, entendendo que uma boa gestão educacional garante o sucesso de toda a comunidade. Portanto, o objetivo deste estudo é elucidar o impacto que um bom planejamento estratégico tem na melhoria do desempenho dos professores no Peru, levando em consideração: suas características, benefícios e processos. Para tanto, foi realizada uma revisão sistemática exploratória e análise dos dados. Por fim, graças à seleção rigorosa das informações, foi possível concluir que, de fato, existe uma relação diretamente proporcional entre o planejamento estratégico e o desempenho docente, expressa na melhoria do tempo, da ordem e da execução.

Palavras chaves: Estratégias educacionais; Planejamento; Qualificações ocupacionais; Gestão; Aprendendo 
INTRODUCCIÓN

Son múltiples las investigaciones que se han orientado a evidenciar las falencias dentro de la gestión educativa, debido al papel que juega en la generación de una educación de calidad que responda a las demandas, necesidades y exigencias de la sociedad. Incluso la Unesco (2020) ha señalado la importancia de contribuir con las autoridades nacionales para implementar políticas educativas que cumplan con los estándares internacionales y desafíos hacia el 2030, siendo uno de los lemas "transformar los compromisos en actos", por lo que la gestión es la vía que llevará a concretar todos los objetivos planteados dentro y fuera de nuestro territorio.

En este sentido, una institución de éxito se caracteriza por tener una planificación estratégica acorde a sus necesidades y demandas, es decir, que le permita mejorar sus procesos y la optimizar los aprendizajes. Por ello, es importante que los actores educativos tengan presente la relevancia de la planeación estratégica, considerada como: un conjunto de procesos de diseño, desarrollo y operación de métodos de intervención que permiten alcanzar las metas y las competencias institucionales establecidas en referencia a las demandas y las oportunidades sociales (Villalón, 2018).

Según Yuquilema et al. (2018) gracias a la planificación estrategia las instituciones educativas se desenvuelven ordenadamente, permitiendo establecer: propósitos, objetivos, políticas y estrategias. Así, ella asegura una eficiente gestión, ya que vela por el cumplimiento de metas y objetivos, previamente establecidos, que determinan la manera en la que se ha de organizar cada una de las actividades previstas. Además, la planificación estratégica influye en la calidad del servicio ofrecido a los estudiantes y padres de familia, por lo que se hace indispensable el uso de una herramienta de gestión para optimizar el servicio educativo, necesariamente vinculado al desempeño docente.

Por otro lado, según la Ley 28044 el docente es un actor importante dentro del proceso educativo, en la medida que se encarga de brindar una formación integral y óptima a los estudiantes. Para llevar a cabo esta misión es necesario que el maestro sea un profesional con: competencia, solvencia moral, salud física y mental, ya que dichas características posibilitan ejercer los desempeños indicados en el Marco del Buen Desempeño Docente (MINEDU, 2012).

El desempeño docente hace referencia a la labor profesional que ejerce el maestro dentro de la institución educativa, cuya finalidad es lograr que los estudiantes adquieran y consoliden sus aprendizajes (Azañedo, 2021). Al respecto, la MINEDU (2012) afirma que las actuaciones del maestro pueden ser descritas y evaluadas para establecer su nivel de competencia. Adicionalmente, El Proyecto Educativo Nacional al 2036 (MINEDU, 2020) resalta la gran importancia del rol docente señalando que este debe ser capaz de hacer que los estudiantes logren lo que se espera, es decir, un aprendizaje autónomo y autorregulado; sin embargo, nada sería posible si no se brindan mejores condiciones estatales para lograr dichos objetivos.

En efecto, la relación entre planificación estratégica y desempeño docente se ha evidenciado en diversos estudios como el de Altuve (2016) que concluye que a niveles moderados el desarrollo efectivo de la Formación Docente influye en el nivel de eficiencia de la Planificación Pedagógica. Del mismo modo, Morante et al. (2018) realizó un proyecto de investigación sobre lo favorable que es 
el uso de la planificación estratégica en los procesos de formación mediados por las TIC, siendo enfático en la proliferación de la planificación hacia áreas no empresariales. También señaló la flexibilidad que ha tenido para adaptarse a las TIC y diversos retos de la globalización.

Por su parte, Diaz et al. (2020) afirma que los efectos de la aplicación de un planeamiento estratégico son diversos: en primer lugar, colabora en la generación de profesionales integrales; en segundo lugar, aumenta la calidad de los servicios y contribuye a la funcionalidad institucional; en tercer lugar, ayuda en la instrucción y superación de los trabajadores; en cuarto lugar, emplea las TIC en aras de incrementar la innovación y gestión; por último, repercute en la sociedad por medio de la mejora de los procesos de aprendizaje que forman integralmente a sus alumnos.

En ese sentido, una de las responsabilidades que el Estado debe de asumir es proporcionar una educación de calidad que requiere de una buena gestión y administración del capital económico y humano. Por ello, es preciso planificar de manera estratégica y articulada a corto, mediano y largo plazo, sin olvidar el objetivo general del MINEDU, es decir, velar por una formación integral que permita el desarrollo de competencias que hagan de los estudiantes mejores personas para que alcancen el éxito profesional en un mundo atravesado por la digitalización y globalización.

Sin embargo, en la mayoría de instituciones educativas, a causa del poco interés en la investigación e innovación, no existen planes estratégicos ni recursos, en otras palabras, a nivel nacional nos enfrentamos al gran problema de organizar la gestión educativa sin una planificación estratégica, siendo su principal consecuencia la falta del cumplimiento de objetivos institucionales. Así, se evidencia que la mayoría de dificultades presentes en las instituciones educativa están relacionadas con su desempeño y la falta de una planeación educativa, fundamentalmente, por la carencia de información técnica y operativa de los directivos.

Lo anterior, proporciona un panorama de reflexión exhaustiva, ya que refleja que las limitaciones con las que cuentan los maestros para ejercer satisfactoriamente su quehacer profesional son: la falta de apoyo organizacional, la falta de apoyo de la directiva del colegio, la falta de interés estatal, etc., lo peculiar es que son los directivos institucionales quienes suelen desplazar a los docentes, excluyéndolos de las reuniones en donde se diseña la planificación educativa, lo cual es perjudicial porque son los docentes los que vivencian las carencias institucionales y poseen conciencia de los verdaderos problemas que aquejan la labor docente y, por ende, las posibles soluciones o respuestas a dichas situaciones.

Esto con el propósito de manifestar una mejora del tiempo, orden y ejecución de las tareas docentes en las instituciones educativas.

\section{MÉTODO}

Para el desarrollo del estudio se realizó un análisis amparada en la metodología de la revisión sistemática exploratoria, con la finalidad de realizar un análisis exhaustivo de la documentación existente sobre la relación entre el proceso de planificación estratégica en Perú y su alcance en el desempeño docente.

El aparataje epistemológico que rige la recolección de fuentes y análisis de información de la presente investigación es la revisión sistemática 
exploratoria, caracterizada por ser eficaz a la hora de seleccionar fuentes de manera clara y ordenada, sin dejar de mencionar el aporte a nivel reflexivo y extensivo, en la medida que no solo actualiza las producciones generadas hasta el momento sobre un fenómeno, sino que permite un amplio marco reflexivo de los alcances de los estudios encontrados (Begoña et al., 2018).

Asimismo, los criterios de inclusión de información se han plasmado en ítems que toman en consideración el año de publicación, las variables como expresiones de búsqueda, solo publicaciones en español e inglés, geolocalización extendida $\mathrm{y}$ empleo de fuentes encontradas en bases de datos reconocidas. Cabe señalar que también se emplearon criterios de exclusión como: descartar los documentos que no se encontraban en bases de datos reconocidas internacionalmente, excluir los escritos que no pasaron la prueba de réplica y dejar de lado aquellas investigaciones que no cumplieran con el rango de años establecidos.

Así, en cuanto al lapso de tiempo considerado, las investigaciones recolectadas se han producido en los últimos 6 años, es decir, desde el 2015 al 2021, salvo aquellos documentos administrativos que constituyen fuentes consultivas a nivel nacional. En el caso de las expresiones de búsqueda, estas se establecieron en torno a las expresiones planificación estratégica y desempeño docente, correspondientes a las variables de la investigación. Adicionalmente, se seleccionaron los siguientes buscadores Elsevier, Science, Direct, Redalyc y Reserchgate, siendo Science y Redalyc las bases de datos con mayores resultados por variables.

Finalmente, para el análisis de la información se clasifico en dos partes. En primer lugar, se buscó la expresión "La planificación estratégica como mejora del desempeño docente en el Perú", gracias a la que se obtuvieron una cantidad mínima de artículos que, en su mayoría, no guardaban relación con el objeto de estudio. En segundo, se emplearon las variables de investigación Planificación estratégica y desempeño docente, gracias a las que se obtuvieron 165 documentos sometidos a una prueba de réplicas, cabe señalar que 75 de los escritos están vinculados a la primera variable y 90 a la segunda.

\section{DESARROLLO Y DISCUSIÓN}

La planificación estratégica tiene un largo devenir histórico, según Basurto (2016) se puede evidenciar desde la edad antigua, en las primeras civilizaciones, como Grecia y Roma, debido a que en estas culturas se ejecutaban planes de acción elaborados mediante el análisis de causas y consecuencias de hechos anteriores, con el fin de poder tener un buen gobierno que permitiera aprovechar las oportunidades del ambiente, es decir, el medio natural y social.

Sin embargo, es a partir del siglo XX que la expresión empieza a utilizarse en diversos ámbitos, siendo Taylor y Fayol quienes impulsan este proceso en la administración al establecer las siguientes dimensiones: planear, organizar, dirigir, coordinar y controlar (Ortiz, 2017). En la actualidad, la gestión escolar también trabaja con la planificación estratégica, siendo su objetivo el hacer que sus instituciones aprovechen al máximo sus recursos y mejoren sus procesos. Así, el éxito de la praxis docente no solo radica en la capacidad de los profesores en transmitir conocimientos, sino en una planeación fundamentada en la reflexión pedagógica (Yautenzi, 2019). 
La planificación estratégica ha ido evolucionando a lo largo del tiempo, por lo que su conceptualización es diversa y se vincula a caracterizaciones sobre la disciplina en la que se encuentran. Según Ortiz, Méndez y Sánchez (2017) tienen razón al afirmar que este tipo de planificación es un proceso flexible que analiza diversas formas de acción futura, tomando en cuenta sus beneficios y perjuicios, con la finalidad de elegir correctamente decisiones presentes; para ello, es necesario cuestionaros lo siguiente: ¿dónde estamos?, ¿a dónde vamos? ¿a dónde deberíamos de ir?

Por su parte, Yautentzi (2019) menciona que la planificación estratégica es "una herramienta de cambio organizacional que requiere de una estructura, un sistema de comunicación, sustentabilidad, flexibilidad y entidad en sus proyecciones, concentración, participación en su visión y aplicación, así como flexibilidad y evaluación" (p.4). Equívocamente esta actividad se ha vinculado al director educativo; sin embargo, es una labor compartida, como lo señala el art. 55 y art.56 de la ley $\mathrm{N}^{\circ} 28044$. Así, mientras los docentes se encargan de planear el procedimiento que seguirán en sus sesiones de clases, los directivos velan por el cumplimiento de la planeación administrativa (Congreso de la República, 2003).

Por otro lado, Chiavenato (2016) considera que los aportes de la planificación estratégica se deben a sus características, relacionadas con: a) Ser sistemáticas, es el comportamiento general de la organización; b) Enfocarse en el futuro, visión general de la organización; c)Crear valor, conjunto de conductas motivadas y orientadas a la ejecución de objetivos concretos; d) Ser participativa, comprensión y participación de los integrantes de la organización en la planeación estratégica; e) Tener continuidad, la planeación debe ser permanente; f) Ser implementada, realización de la planeación estratégica, exige conocimiento y compromiso por parte de todos los miembros y $G$ ) ser monitoreada, el desempeño y los resultados de la planeación estratégica deben ser evaluados.

Sin embargo, cuando una institución trabaja bajo este tipo de planificación debe considerar que su puesta en práctica no solo depende de sus cualidades o características, sino del compromiso que asuman los integrantes de la organización, conscientes de su importancia y conocedores del proceso de ejecución. Por lo que es sumamente importante que todos se encuentren a la vanguardia, ya que no es posible realizar procedimientos que se desconocen.

Por otro lado, Yuquilema (2018) considera que las etapas de la planificación estratégica son: en primer lugar, la Formulación delas estrategias o etapa en la que se formulan los objetivos en prospectiva; en segundo lugar, la Implementación de estrategias, con la finalidad de mejorar la estructura y, por ende, los resultados de la oferta que se proporciona a la sociedad; por último, la evaluación de la estrategia, entendida como la etapa en la que se refuerzan o corrigen las tácticas y mecanismos implementadas por la organización, para ello es necesario un examen riguroso de los factores internos y externos.

En efecto, la planificación estratégica es un conjunto de acciones que permiten una proyección a futuro por medio del establecimiento de objetivos que vinculan a todos los miembros de una institución. En el ámbito educativo, se asocia al buen desempeño docente, cotidianamente concebido 
como: aquel en donde existe una preocupación por el desarrollo integral de los estudiantes, ya que consciente de las múltiples realidades de los educandos se agencia de los recursos necesarios para lograr que se desenvuelvan eficazmente frente a los problemas u obstáculos de la realidad (Martínez, 2020).

Desde la perspectiva estatal, el Marco del Buen Desempeño Docente (MINEDU, 2012) afirma que el desempeño docente se mide en torno a tres aspectos: 1) Actuación observable, esta puede ser descrita o medida; 2) Responsabilidad, cumplimiento de las funciones de la profesión docente y 3) Logro de determinados resultados, determinación de logros generales y específicos que el docente ha alcanzado en su actuación profesional.

Por su parte, Valdés (2005) sostiene que la evaluación del desempeño docente debe comprender la realización de las siguientes funciones: en primer lugar, el diagnóstico, implica la identificación de las fortalezas y falencias de la instituciones; en segundo lugar, la instrucción, alude a los indicadores obtenidos por los docentes al desarrollar una autoevaluación; en tercer lugar, la educación,es el perfeccionamiento de la función docente, a partir del perfeccionamiento de sus habilidades; por último, la función desarrolladora, complementa la búsqueda de perfeccionamiento docente, a causa de la constancia evaluativa.

En efecto, son diversos los criterios que se asumen a la hora de determinar un desempeño docente eficaz; sin embargo, ello no debe menoscabar el indiscutible esfuerzo que realizan los maestros para garantizar el proceso de enseñanza y aprendizaje. Por ello, en el ámbito de la planeación estratégica son los canalizadores de los objetivos institucionales que, en primera instancia, responden al desarrollo del potencial de sus estudiantes. Así, no se trata solo de cuantificar el lugar que tiene el docente en una institución educativa, también se debe considerar que el ejercicio profesional del docente complica o disminuye su rendimiento debido a diversos factores como los señalados por Escribano (2018) falta del reconocimiento social y estatal, de tiempo libre que permita ahondar en la investigación y perfeccionamiento de su labor, de acceso a capacitaciones que permitan actualizar las estrategias y metodologías a emplear.

Por lo mencionado, son diversas las investigaciones que señalan que los maestros podrían mejorar su desempeño apelando a la planificación estratégica en su práctica profesional, con el fin de optimizar sus procesos en el aula. Ello, debido a que la planificación estratégica es un conjunto de acciones y decisiones que los docentes implementan durante el ejercicio de su trabajo profesional tomando en cuenta su carga horaria, la temática, criterios y procesos de evaluación. En efecto, una mejor distribución y delimitación de la práctica pedagógica puede asegura que todos los estudiantes tengan las mismas oportunidades de desarrollar sus competencias (Ramírez, 2019).

En cierta medida, el MINEDU (2012) postula algo similar al afirmar que la escuela debe aspirar a tener: 1) Gestión escolar, dirección que asuma un liderazgo pedagógico que asuma el generar estrategias que propicien los logros estudiantiles; 2) Convivencia, establecimiento de un espacio inclusivo, acogedor y colaborador; 3) Relación escuela-familia-comunidad, incentivar el compromiso de los agentes del entorno estudiantil en la adecuación y logro de los aprendizajes de 
los mismos y 4) Procesos pedagógicos, actividad docente por la que se genera un aprendizaje crítico, ingenioso y profundo.

Por su parte, Ramírez (2019) indica en su estudio que esta estructura de planificación puede ser aprovechada por los docentes en su labor pedagógica, pero añade que esto "constituye un modelo o patrón que permite al docente enfrentar su práctica educativa de forma ordenada y congruente" (p. 8). Asimismo, León (2016) sostiene que el docente actual debe desarrollar planes estratégicos que le permitan mejorar su desempeño profesional e institucional, ya que la planeación anual de un curso no tiene por qué ser incongruente con la planificación general (p. 34).

Porúltimo, dentro del desarrollode planificación estratégica para el desempeño docente, se debe tomar en cuenta tres intereses: "individuales del docente, institucionales de la escuela y propias del sistema educativo en su conjunto; de este modo, se logrará el desarrollo profesional del maestro y el mejoramiento de la calidad de la enseñanza" (Lombardi y Abrile, citado por León, 2016, p. 34). En efecto, a pesar de la multiplicidad de investigaciones todas coinciden en afirmar que una adecuada planificación tiene como resultado un desempeño docente exitoso.

\section{CONCLUSIÓN}

En base a la literatura revisada, se concluye que la planificación estratégica es la acción que permite al docente desarrollar su propuesta de clase pedagógica; por ello, se la considera como un trabajo propio e inherente al maestro. Esta es considerada como un instrumento que facilita su trabajo, buscando delimitar los fines, objetivos y metas de la educación. Así mismo, define qué hacer, cómo hacerlo y qué recursos y estrategias utilizar para alcanzar los objetivos propuestos por el docente en su propuesta de sesión de aprendizaje. De esta manera, se reduce la incertidumbre y se anticipa a las diversas situaciones que puedan surgir en el desarrollo de las sesiones.

Para que la planificación estratégica sea eficiente se deberá ejecutar con responsabilidad, después de haber monitoreado los procesos implementados, teniendo en cuenta el control y evaluación de los mismos. De este modo, se aprovecharán sus ventajas y fortalezas, superando las limitaciones que se puedan encontrar dentro del proceso de enseñanza y aprendizaje en la que está inmersa la labor del docente, ya que una buena labor docente no solo es el resultado de tener gran conocimiento en el tema de especialización y en saberlo compartir con los estudiantes, también se expresa en el diseño de una buena planificación estratégica realizada bajo el análisis del contexto donde se encuentre la institución educativa y los estudiantes, el trabajo colaborativo de pares y la reflexión del actuar pedagógico.

REFERENCIAS

Altuve, D. (2016). La formación docente en el proceso de la planificación pedagógica. Educación en Contexto, 2, 129 - 145

Azañedo (2021). El desempeño docente antes y durante la pandemia. Polo del Conocimiento, 6(4). https://polodelconocimiento.com/ojs/ index.php/es/article/view/2614

Basurto, X. (2016). Un acercamiento de la planificación estratégica y las organizaciones sanitarias. Revista científica dominio de las ciencias, 2(3), 3 - 14. https:// dominiodelasciencias.com/ojs/index.php/es/ article/view/109 
Chiavenato, I. (2016). Planificación estratégica. Fundamentos $y$ aplicaciones. https:// www.remax-accion.com.ar/wp-content/ uploads/2021/04/127-Planeacion-estrategicafundametos-chiavenato-idalberto.pdf

Congreso de la República del Perú. (2003, 29 de julio). Ley $\mathrm{N}^{\circ}$ 28044. Ley General de Educación. Diario Oficial El Peruano. https://www.gob.pe/ institucion/congreso-de-la-republica/normaslegales/118378-28044

Diaz, M., Alarcón, R. y Saborido, J. (2020). Potencial humano, innovación y desarrollo en la planificación estratégica de la educación superior cubana 2012 - 2020. Revista Cubana de Educación Superior, 39(3). http://scielo.sld.cu/ pdf/rces/v39n3/0257-4314-rces-39-03-e1.pdf

Escribano, E. (2018). El desempeño del docente como factor asociado a la calidad educativa en América Latina. Revista Educación, 42(2), 1-25. https:// www.redalyc.org/articulo.oa?id=44055139021

León, H. (2016). Gestión de la planificación estratégica para el desarrollo profesional docente. Rev. Gestión de la Educación, 6(1), 23 52. https://revistas.ucr.ac.cr/index.php/gestedu/ article/view/22719/22925

Martínez, G., Esparza, A. y Gómez, R. (2020). El desempeño docente desde la perspectiva de la práctica profesional. RIDE. Revista Iberoamericana para la Investigación y el Desarrollo Educativo, 11(21), e013. Epub 09 de marzo de 2021.https://doi.org/10.23913/ride. v11i21.703

MINEDU (2012). Marco del Buen Desempeño Docente. http://www.MINEDU.gob.pe/pdf/ed/ marco-de-buen-desempeno-docente.pdf

MINEDU (2020). Proyecto Educativo Nacional PEN 2036 -. https://www.cne.gob.pe/uploads/ publicaciones $/ 2020 /$ proyecto-educativonacional-al-2036.pdf

Morante, G., Dugarte, E. y Herrera, J. (2019). Perfil del aprendiz estratégico para el estudio de Cálculo Diferencial mediado por las TIC. Revista Logos Ciencia y Tecnología, 11(3). https://n9.cl/yzda4
Ortiz, M. (2017). Breve reseña acerca de la planificación estratégica. Revista multidisciplinar de innovación y estudios aplicados, 2(4), p. 188 - 197. https://redib.org/Record/oai_ articulo2287615-breve-rese $\% \mathrm{C3} \% \mathrm{~B} 1 \mathrm{a}$-acercade-la-planeaci\%C3\%B3n-estrat\%C3\%A9gica

Ramírez, J. R. (2019). La planeación estratégica y la calidad docente en la Universidad. Revista Torreón Universitario, 7(19), 6-17. https://doi. org/10.5377/torreon.v7i19.7906

Unesco (2020). Unesco Covid - 19. Education Response. 1 https://en.unesco.org/covid19/ educationresponse

Valdés, V. (2005). Evaluación del Desempeño docente. Ponencia presentada por Cuba. Encuentro Iberoamericano sobre Evaluación del Desempeño docente. Ciudad de México, 23 al 25 de mayo

Villalón, E. (2018). Planeación estratégica en la escuela. Revista Vinculando. https://vinculando. org/educacion/planeacion-estrategica-en-laescuela.html

Yautentzi, D. (2019). La planeación estratégica como herramienta para diseñar proyectos transversales en educación y su impacto en la evaluación. Debates en Evaluación y Curriculum. https://posgradoeducacionuatx.org/pdf2018/ A097.pdf

Yuquilema, M., Ilvis, J. y Quishpi, V. (2018). Plan estratégico institucional para el mejoramiento administrativo, técnico y pedagógico de las unidades educativas. http://142.93.18.15:8080/ jspui/bitstream/123456789/219/1/PLAN\%20 ESTRATEGIC O \% 20 E D U C A CION \% 20 INTERCULTURAL\%20BILINGUEB.pdf 